\title{
Theranostics
}

$2011 ; 1: 354-362$

Research Paper

\section{Multimodal Bacteriochlorophyll Theranostic Agent}

\section{Tracy W.B. Liu ${ }^{1,2}$, Juan Chen², Laura Burgess ${ }^{1,2}$, Weiguo Cao ${ }^{1,3}$, Jiyun Shi, ${ }^{1,2}$ Brian C. Wilson ${ }^{1,2}$ and Gang Zheng ${ }^{1,2, \square}$}

1. Department of Medical Biophysics, University of Toronto, Toronto, Canada;

2. Ontario Cancer Institute, University Health Network, Toronto, Canada;

3. Department of Chemistry, Shanghai University, Shanghai, China

$\triangle$ Corresponding author: Gang Zheng, PhD, University of Toronto, 101 College Street, TMDT 5-363, Toronto, ON M5G1L7, Canada. Tel: 416-581-7666; Fax: 416-581-7667; E-mail: gang.zheng@uhnres.utoronto.ca

(C) Ivyspring International Publisher. This is an open-access article distributed under the terms of the Creative Commons License (http://creativecommons.org/ licenses/by-nc-nd/3.0/). Reproduction is permitted for personal, noncommercial use, provided that the article is in whole, unmodified, and properly cited.

Received: 2011.07.23; Accepted: 2011.07.30; Published: 2011.08.23

\begin{abstract}
The complimentary ability of different noninvasive imaging technologies with therapeutic modalities can be used in tandem providing high-resolution and highly sensitive imaging of events at the molecular and cellular level providing a means for image-guided therapy. There is increasing interest in using porphyrin-based photosensitizers as theranostics to take advantages of their near-infrared fluorescent properties for imaging and their strong singlet oxygen generation abilities for photodynamic therapy. Here we report a targeted multimodal bacteriochlorophyll theranostic probe. This probe consists of a bacteriochlorophyll derivative, a pharmacokinetics modification peptide linker and folate for targeted delivery to folate receptor expressing cancer cells. We demonstrate its multimodal theranostic capability, its folate receptor targeting ability and its utility for both NIR fluorescence imaging and photodynamic therapy purposes both in vitro and in vivo.
\end{abstract}

Key words: Photodynamic therapy, Fluorescence, Porphyrin, Folate receptor and peptides

\section{Introduction}

The idea of using multiple modalities in conjunction in the field of oncology has come into fruition over the past decade. The complimentary ability of different non-invasive imaging technologies with therapeutic modalities can be used in tandem providing high-resolution and highly sensitive imaging of events at the molecular and cellular level providing a means for image-guided therapy, also known as theranostics [1]. For example, fluorescence is exploited as a possible technique for image-guided surgical resection. Optical imaging provides real-time information about surgical margins, thereby, extending the surgeon's vision ensuring complete surgical resection of tumors [2-3]. To increase the contrast of malignant tissues, probe development with high specificity, selectivity and stability is highly desirable for the imaging of disease. Furthermore, the field of photodynamic therapy (PDT) has seen an insurgence of fluorescence guided PDT due to the inherent multifunctional nature of some photosensitizers (PS) which produce both fluorescence emission and singlet oxygen when excited [4-7]. PDT is a clinically approved cancer treatment combining light-activated drugs known as PS, light and oxygen [8-9]. Consequently, tumor destruction results from the production of cytotoxic singlet oxygen [8-9]. PS development is important as accumulation of PS dictates which tissues are destroyed, thus, by developing PS that better concentrate within target tissue, we increase the contrast within target tissue, increasing our ability to 
detect and treat lesions while preserving healthy tissue during therapy. There is increasing interest in using porphyrin-based molecules in PDT which is advantageous as they are bifunctional compounds with near-infrared fluorescent properties and efficacious PS for PDT [10-13].

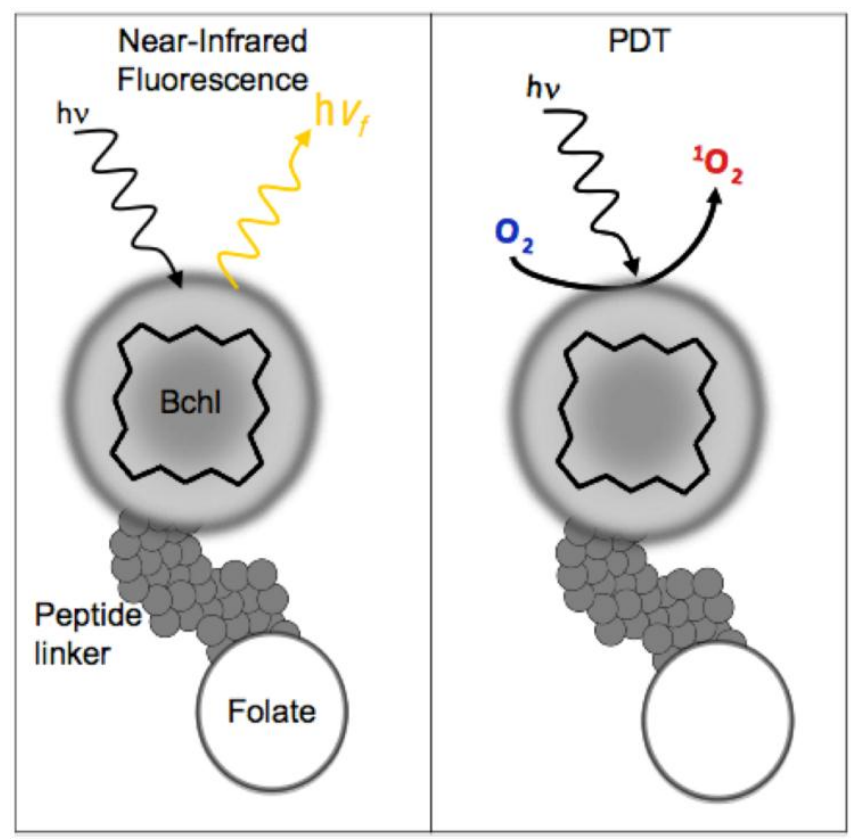

Figure I. BPF schematic. There are three components to BPF: Multifunctional Bchl, a peptide linker and a targeting moiety (Folate). BPF is multi-modal: I) Near-infrared fluorescence imaging and 2) PDT.

Bacteriochlorophyll (Bchl) is emerging as a powerful multifunctional porphyrin. Bchl is extracted from R. Sphaeroids and is the most widely distributed bacteriochlorin pigment [14]. It is a near-infrared PS with 750-850 nm excitation and emission profile making it highly attractive for optical imaging [15]. Near-infrared wavelengths $(650-900 \mathrm{~nm})$ are optimal for fluorescence imaging as they providedeeper tissue penetration due to the low tissue absorption and low autofluorescence resulting in higher signal-to-noise ratios [2]. Despite its favorable characteristics, early Bchl studies demonstrated limited application in PDT and optical imaging due to its unstable nature. Many efforts have occurred to address the instability of Bchl including modification of the isocyclic ring [14, 16], serine stabilization [17], fluorinated stabilization [18], inserting palladium to form a more stable complex (TOOKAD) [19] and incorporation into nanoparticles [15][20]. Regardless of the stabilization technique,
Bchl has established itself as an ideal bifunctional imaging and PDT agent, in particular, palladium-Bchl (TOOKAD) has been used in patients for PDT treatment of prostate cancer [21-22]. We report here the first targeted multimodal Bchl theranostic probe (BPF) that follows a similar strategy to our previously reported pyropheophorbide-peptide-folate (PPF) probe [23]. Like PPF, BPF consists of Bchl, a pharmacokinetics modification peptide linker [23] and folate for targeted delivery to FR-expressing cancer cells (Figure $1)$. The key difference between BPF and PPF is that BPF is significantly red-shifted $\left(\lambda_{\text {excitation }}=748 \mathrm{~nm}\right.$ and $\left.\lambda_{\text {emission }}=766 \mathrm{~nm}\right)$, making it ideally suited for near-infrared imaging of deeper seeded tumors. Here we demonstrate the multimodal theranostic capability of BPF, its FR targeting capability and its utility for both NIR fluorescence imaging and photodynamic therapy purposes.

\section{Methods and Materials}

General Materials: The activating agents 1-hydroxybenzotriazole (HOBt) and (benzotriazol-1-yl)-N,N,N',N'-tetramethyluronium hexafluorophosphate (HBTU) were purchased from Sigma-Aldrich and Novabiochem, and used without further purification. The Rink amide resins and all the 9H-fluoren-9-ylmethoxycarbonyl ( $\mathrm{N}-\alpha$-Fmoc)-protected amino acids were purchased from Novabiochem. Bacteriopheophorbide a (Bchl acid) and folate succinimide (Folate-NHS) were synthesized by the previous described protocols $[15,23]$.

General HPLC Methods: Reverse-phase analytical high performance liquid chromatography (HPLC) experiments were performed on a XBridge-C18 column $(2.5 \mu \mathrm{m}, 4.6 \mathrm{~mm} \times 50 \mathrm{~mm})$ using a Waters 2695 controller with a 2996 photodiode array detector and a Waters ZQ mass detector. The conditions were as follows: solvent A) acetonitrile; solvent B) $0.1 \%$ trifluoroacetic acid (TFA); gradient, from $20 \% \mathrm{~A}+80 \% \mathrm{~B}$ to $100 \% \mathrm{~A}+0 \% \mathrm{~B}$ in $12 \mathrm{~min}$; flow rate, $0.8 \mathrm{~mL} \mathrm{~min}^{-1}$.

Synthesis of BPF: A peptide sequence with D amino acid backbone, Fmoc-gd(OtBu)e(OtBu)vd $(\mathrm{OtBu}) \mathrm{gs}(\mathrm{tBu}) \mathrm{gk}(\mathrm{Mtt})$, was synthesized on Rink resin using Fmoc chemistry protocol. After removing the last Fmoc group, Bchl acid was coupled to the $\mathrm{N}$-terminal of the peptide on resin at room temperature ([Bchl acid/HOBt/HBTU/peptide 3:3:3:1]). The Bchl-peptide-resin was then treated with a cleavage cocktail (TFA: triisopropylsilane: water $=95: 2.5: 2.5$ ) for $1 \mathrm{~h}$ at room temperature to remove the resin and cleave the protected groups. The acquired Bchl-peptide (BP) was divided into two parts. One part was purified by HPLC and used as a folate-free control in following studies. The other part was con- 
jugated with folate-NHS according to the previously reported protocol [23]. The acquired Bchl-peptidefolate (BPF) was purified by HPLC (Figure 2A).The UV-visible spectrum of BPF was measured using a Varian Cary 50 UV-visible spectrophotometer (Figure $2 \mathrm{~B})$. BPF was prepared in DMSO at a concentration of $1 \mu \mathrm{M}$.

Cell Lines and Culture Conditions: Epithelial carcinoma cells, KB and HT1080, were grown and maintained in Minimum Essential Medium Eagle (MEM) media supplemented with $10 \%$ fetal bovine serum at $37{ }^{\circ} \mathrm{C}$ in an atmosphere of $5 \% \mathrm{CO}_{2}$ in a humidified incubator.

In vitro PDT efficacy: Approximately $2 \times 10^{4}$ cells per well $(200 \mu \mathrm{L})$ were seeded in Nunc Laboratory-TekIICC2 96-multiwell plates and incubated for 2 days at $37{ }^{\circ} \mathrm{C}$ under $5 \% \mathrm{CO}_{2}$. The cell media was changed to folate-free Park Memorial Institute (RPMI) 1640 media $24 \mathrm{~h}$ prior to treatment. BPF $(5 \mu \mathrm{M})$ was dissolved in $2 \%$ DMSO and $0.01 \%$ Tween- 80 in $200 \mathrm{uL}$ of folate-free RPMI 1640 media and incubated with cells for $16 \mathrm{~h}$ at $37{ }^{\circ} \mathrm{C}$ under $5 \% \mathrm{CO}_{2}$. The cells were then rinsed with PBS, resuspended with $150 \mu \mathrm{L}$ of the MEM medium and illuminated by light. The light source consisted of a 740nm light box consisting of 48 LED diodes (Roithner Lasertechnik, Vienna, Austria). The fluence rate was $6.3 \mathrm{~mW} / \mathrm{cm}^{2}$. Cell viability was then determined by means of the colorimetric MTT assay. Briefly, after illumination, the cells were incubated at $37{ }^{\circ} \mathrm{C}$ under $5 \% \mathrm{CO}_{2}$ for $24 \mathrm{~h}$. The medium was removed and 3-(4,5-dimethylthiazol-2-yl)-2,5diphenyltetrazolium bromide (Invitrogen) solution in medium $(0.5 \mathrm{mg} / \mathrm{mL}, 150 \mu \mathrm{L})$ was added to each well followed by incubation for $2 \mathrm{~h}$ under the same environment. $150 \mu \mathrm{L}$ of a 1:1 ratio of DMSO to $70 \%$ isopropanol in $0.1 \mathrm{M} \mathrm{HCl}(10 \%$ by weight, $100 \mu \mathrm{L})$ was then added to each well. The plate was agitated on a Spectra Max Plus microplate reader (Molecular Devices Corporation) for $5 \mathrm{~s}$ before the absorbance at $570 \mathrm{~nm}$ at each well was taken.

In vivo model: All animal studies were carried out under institutional approval (University Health Network, Toronto, Canada). Adult athymic female nude mice were inoculated subcutaneously with $2 x$ $10^{6} \mathrm{~KB}$ or HT1080 cells in $200 \mu \mathrm{L}$ of PBS media on the right or left flank under general anesthesia (isofluorane in oxygen). Animals were maintained in pathogen-free conditions in autoclaved microisolator cages in the MaRS Animal Resource Centre.

In vivo optical imaging studies: Mice bearing KB (right flank) and HT1080 (left flank) tumors were used to compare the tumor uptake and folate receptor targeting capability of BPF versus BP. $25 \mathrm{nmol}$ of BPF or $\mathrm{BP}$ was formulated in $150 \mu \mathrm{L}$ of aqueous solution using $5 \mu \mathrm{L}$ of DMSO and $1.5 \mu \mathrm{L}$ of Tween 80 . When the tumor size reached $5-10 \mathrm{~mm}$ in diameter, mice were intravenously injected via tail vein with $\mathrm{BPF}(\mathrm{n}=5)$ or BP $(n=5)$ under general anesthesia. Whole-body in vivo fluorescent imaging was performed before and at multiple time points (10min, $3 \mathrm{~h}, 5 \mathrm{~h}, 24 \mathrm{~h}$ and $48 \mathrm{~h}$ ) after injection (Maestro ${ }^{\mathrm{TM}}$, CRi: $680 \mathrm{~nm}$ excitation, $\geq 700 \mathrm{~nm}$ longpass detection, autoexposure integration time, total fluorescence signals normalized by exposure time and ROI area (total signal/(ms * pixels)). Comparison between two different probes was made using the two-sample homoscedastic student $\mathrm{t}$-test with the level of significance set at $p<0.05$. Comparison of probe uptake over time was made using a paired student t-test with the level of significance set at $p$ $<0.05$.

Ex vivo xenograft studies: $\mathrm{KB}$ and $\mathrm{HT} 1080$ tumors were harvested from mice $48 \mathrm{~h}$ following BPF or $\mathrm{BP}$ injection. Tumors were imaged (Maestro ${ }^{\mathrm{TM}}, \mathrm{CRi}$ : $680 \mathrm{~nm}$ excitation, $\geq 700 \mathrm{~nm}$ longpass detection, autoexposure integration time, total fluorescence signals normalized by exposure time and ROI area (fluorescent signal/(ms * pixels)) and weighed in order to assess the tumor uptake of BPF vs. BP in KB vs. HT1080 xenografts. Ex vivo fluorescent signals were corrected for weight. Comparison between two different probes was made using the two-sample homoscedastic student $\mathrm{t}$-test with the level of significance set at $p<0.05$.

In vivo PDT efficacy: Mice bearing KB tumors were intravenously injected with $50 \mathrm{nmol}$ of BPF $(n=5)$ under general anesthesia. BPF uptake was monitored by in vivo fluorescence imaging and $3 \mathrm{~h}$ after injection PDT treatment was given to the tumor. Using a 740nm continuous wave pig tail diode laser, tumors were treated with a single PDT light dose of $137 \mathrm{~J}$ using a surface irradiation of $0.785 \mathrm{~cm}^{2}$ and fluence rate of $75 \mathrm{~mW} / \mathrm{cm}^{2}$. The mice were then monitored daily. A drug-free control animal was treated with the same light conditions without BPF. The control mouse was sacrificed after 7 days as the tumor size had reached experimental endpoint.

\section{Results}

We designed the first prototype targeted Bchl probe based on the following principles: 1) Bchl is an effective imaging and therapeutic agent, 2) the Caspase- 3 peptide sequence with $\mathrm{D}$ amino acid backbone is a stable linker and modulator improving the delivery efficiency of Bchl and 3) folate is an effective homing molecule targeting FR-expressing tumors (Figure 1). On the basis of this design, we have synthesized Bchl-gdevdgsgk(folate) (BPF). The peptide sequence was synthesized following the Fmoc 
solid phase peptide synthesis protocol using a PS3 peptide synthesizer. The Fmoc protecting group was removed by $20 \%$ piperidine in N,N-Dimethylformamide and Bchl acid was conjugated to the $\mathrm{N}$-terminal of the peptide. The Bchl-peptide resin was then treated with a cleavage cocktail to remove the resin and protecting groups allowing the subsequent coupling with folate. The final product was purified by HPLC (Figure 2A) and characterized by UV-visible spectroscopy with special absorption at 357, 527 and $748 \mathrm{~nm}$ (Figure 2B) and ESI mass spectrometry $(\mathrm{m} / \mathrm{z}$ calcd for $\mathrm{C}_{87} \mathrm{H}_{107} \mathrm{~N}_{21} \mathrm{O}_{27}[\mathrm{M}]^{+}$1878.90, found 939.48 $[\mathrm{M}]^{2+}$ and $1879.16[\mathrm{M}]^{+}$, Figure 2C). With the purified BPF product, preliminary in vitro PDT studies were first conducted to evaluate the photocytotoxicity of BPF in FR expressing KB cells. KB cell viability was investigated after $16 \mathrm{~h}$ incubation with $5 \mu \mathrm{M}$ of $\mathrm{BPF}$ in the absence and presence of light (Figure 2D). The formulation used (DMSO and Tween-80) was non-toxic to cells. Using $10 \mathrm{~J} / \mathrm{cm}^{2}$ and $20 \mathrm{~J} / \mathrm{cm}^{2}$ light doses and $5 \mu \mathrm{M}$ and $15 \mu \mathrm{M} \mathrm{BPF}$, a gradient in cell vi-

A

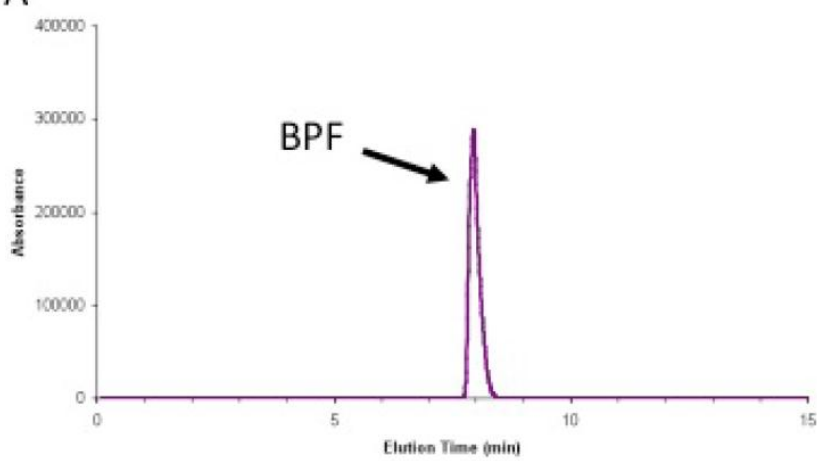

C

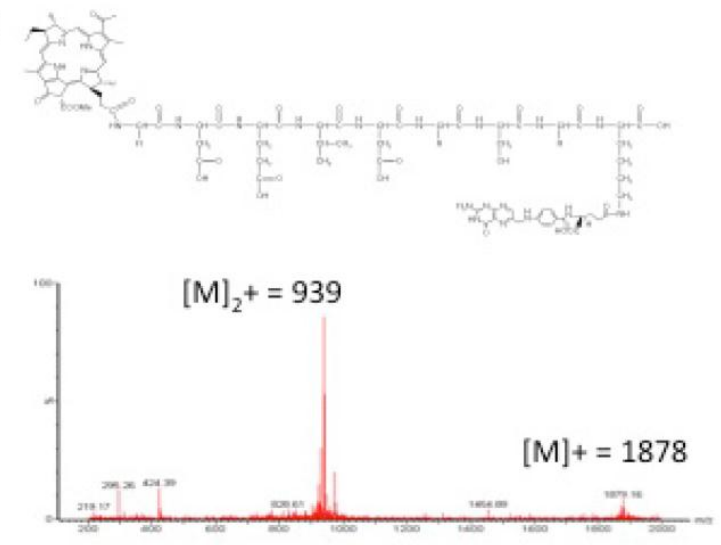

ability was seen dependent on both light dose and BPF concentration as expected (Figure 2D, and Supplementary Material: Figure S1). A BPF concentration of $5 \mu \mathrm{M}$ with a light dose of $10 \mathrm{~J} / \mathrm{cm}^{2}$ reduced cell viability to $70.8 \% \pm 1.9 \%$. When the light dose was increased to $20 \mathrm{~J} / \mathrm{cm}^{2}$, cell viability was reduced to less than $34.7 \% \pm 9.3 \%$. When the concentration of BPF was increased to $15 \mathrm{uM}$, cell viability was reduced to less than $30 \%$ and $5 \%$ for a $10 \mathrm{~J} / \mathrm{cm}^{2}$ and $20 \mathrm{~J} / \mathrm{cm}^{2}$ light dose respectively. This study demonstrates the PDT efficacy of BPF in vitro.

To validate the tumor specificity and FR mediated uptake of BPF, BPF versus BP tumor uptake was evaluated in vivo with nude mice bearing KB (high FR expression) on their right flank and HT1080 (low FR expression) on their left flank. Prior to Bchl probe injection, minimal autofluorescence was seen in the animals. After a $25 \mathrm{nmol}$ intravenous injection of BPF $(1.8 \mathrm{mg} / \mathrm{kg})$ or BP $(1.4 \mathrm{mg} / \mathrm{kg})$, whole body fluorescence was detectable within 10min (Figure 3).

B

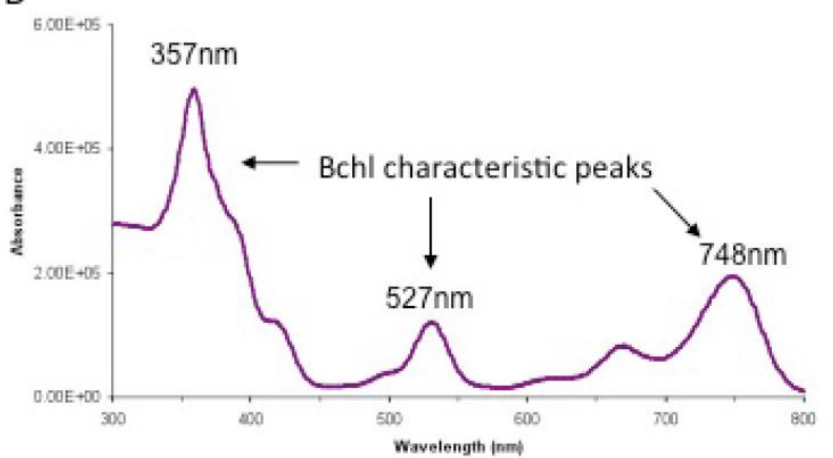

D

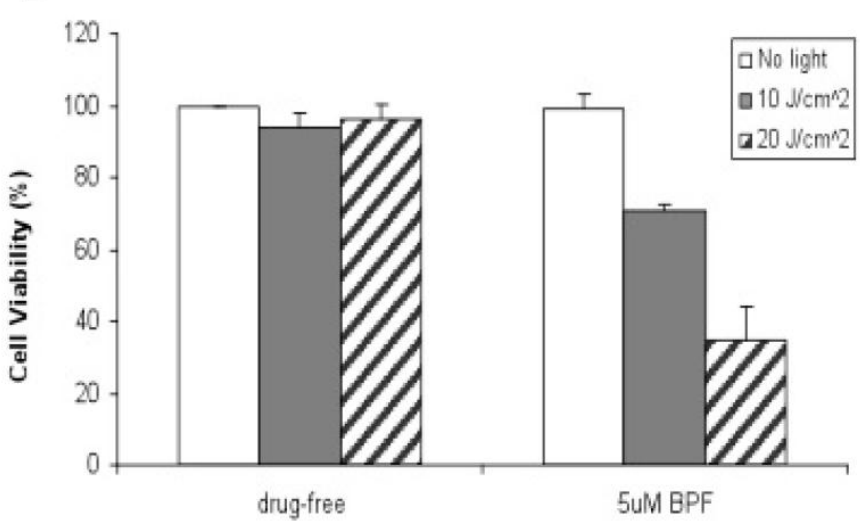

Figure 2. BPF Characterization: A) HPLC chromatogram at 748nm B) UV Absorbance spectra C) Molecular structure and the observed mass spectrum and D) in vitro PDT efficacy of $5 \mu M B P F$ in $K B$ (FR positive) cells using $10 \mathrm{~J} / \mathrm{cm} 2$ and $20 \mathrm{~J} / \mathrm{cm} 2$ light doses. Data are expressed as mean values \pm SEM of three independent experiments, each performed in triplicate. 

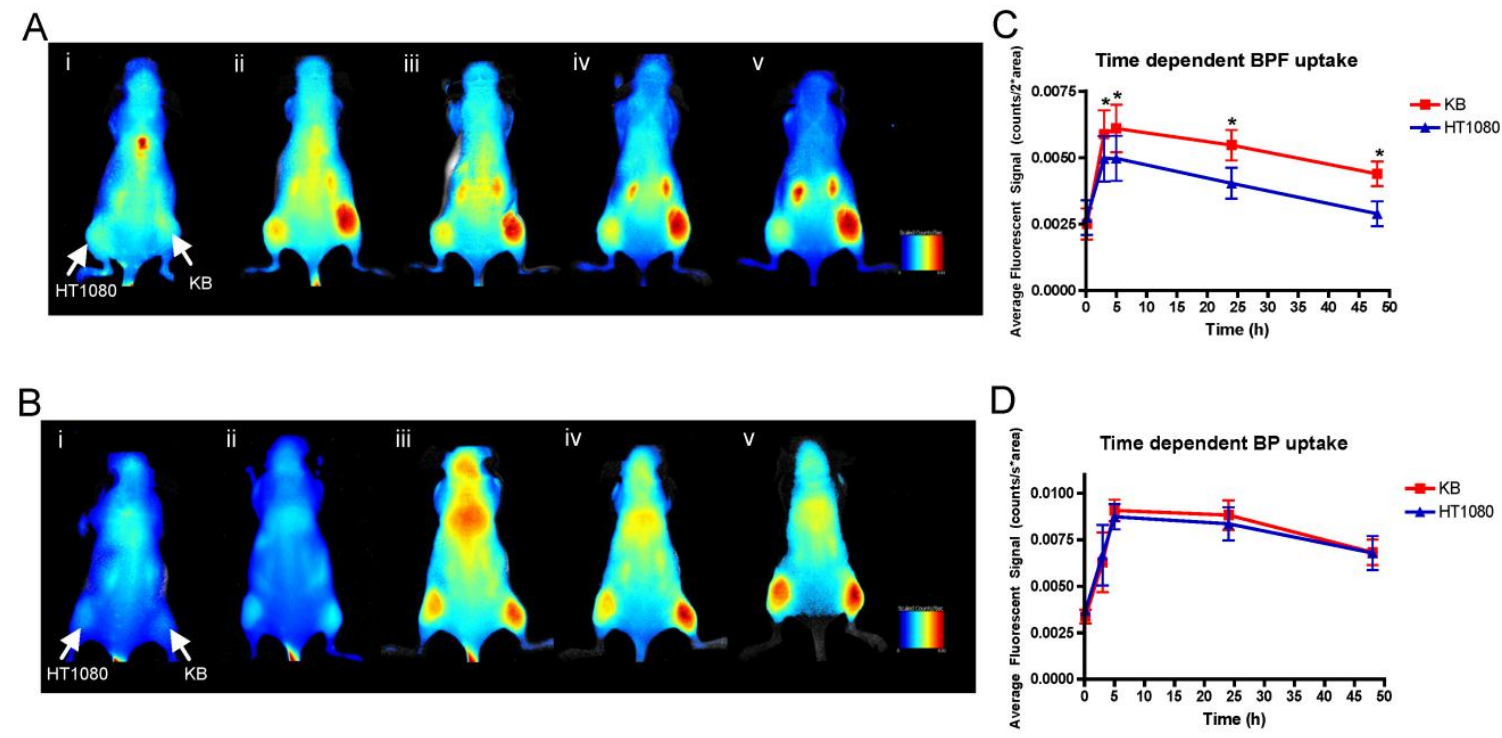

Figure 3. Spectral reflectance fluorescence images of tumor-bearing mice with $K B$ (high $F R$ expressing, right flank) and HTI 080 (low FR expressing, left flank) xenografts. Mice were administered with $25 \mathrm{nmol}$ (in $200 \mu \mathrm{L}$ ) of A) BPF and B) BP and imaged i) $10 \mathrm{~min}$, ii) $3 \mathrm{~h}$, iii) $5 \mathrm{~h}$, iv) $24 \mathrm{~h}$, v) $48 \mathrm{~h}$ post intravenous injection. The in vivo time dependent $\mathrm{KB}$ versus $\mathrm{HTI} 080$ tumor uptake of C) BPF and D) BP is quantified based on the average fluorescent signal (counts/sec*area). Data are expressed as mean values $+\operatorname{SEM}(n=5)$ where * indicates $p<0.05$

Within $3 \mathrm{~h}$, strong fluorescence signal was detectable in $\mathrm{KB}$ tumors in the animals injected with $\mathrm{BPF}$ and remained in $K B$ tumors for $48 \mathrm{~h}$ whereas only a weak fluorescent signal was detectable in HT1080 tumors (Figure 3A and $\mathrm{C}$ ). At all time points for the animals administered $\mathrm{BPF}$, the fluorescent signal in KB tumors was significantly higher $(p<0.05)$ than the fluorescent signal in HT1080 tumors (Figure 3C). Furthermore, the BPF signal in HT1080 xenografts significantly decreased between $5 \mathrm{~h}$ and 24h $(p<0.05)$ while the BPF signal in KB xenografts did not significantly decrease $(p>0.1)$. Animals injected with BP showed strong fluorescence signal in both $\mathrm{KB}$ and HT1080 tumors but not until 5h (Figure 3B and D). BP accumulated in the tumors regardless of their FR expression (Figure 3B and D). BP fluorescent signals significantly decreased $24 \mathrm{~h}$ after injection $(p<0.05)$. After $48 \mathrm{~h}$, animals were euthanized and tumors were harvested. Ex vivo fluorescent imaging of $\mathrm{KB}$ and HT1080 tumors mirror the in vivo imaging. Again, BPF preferentially accumulated in FR expressing $\mathrm{KB}$ tumors significantly greater $(p<0.05)$ than in HT1080 tumors while there is no significant difference in the uptake of BP between KB and HT1080 xenografts (Figure 4). This demonstrates the FR specificity of BPF and the importance of folate as a tumor homing molecule.

The in vivo photocytotoxicity of BPF was then evaluated in mice bearing $K B$ tumors on their right flank. The dose of BPF $(3.6 \mathrm{mg} / \mathrm{kg})$ was increased to twice that used for fluorescence imaging to ensure PDT efficacy, however, this dose of $3.6 \mathrm{mg} / \mathrm{kg}$ is lower than the $5 \mathrm{mg} / \mathrm{kg}$ photosensitizer doses of Bchl analogs (for example, TOOKAD) used in previous xenograft PDT studies [24-26]. Three hours after an intravenous injection of $\mathrm{BPF}$, using a 740nm CW laser at $75 \mathrm{~mW} / \mathrm{cm}^{2}$ with a $1 \mathrm{~cm}$ in diameter surface irradiation, $\mathrm{KB}$ tumors were treated with a total light dose of $137 \mathrm{~J}$. A drug free control animal was treated with the same conditions without the injection of BPF. The treated tumor in animals administered BPF became swollen within $24 \mathrm{~h}$ and within $48 \mathrm{~h}$ signs of tumor necrosis was evident (Figure 5A). All treated tumors in the mice injected with BPF $(n=5)$ reduced in size within two weeks and 30 days after PDT, 3 mice showed complete tumor regression without any signs of tumor regrowth (Figure 5A v and vi) and 2 animals showed small tumor regrowth most likely due to inefficient light treatment as a result of the difficulty in visualizing light at $740 \mathrm{~nm}$ to ensure complete light delivery to the tumor site. In contrast, the tumor on the drug-free control animals continued to increase in size after PDT light treatment. Within 7 days, the tumors were oversized $(1.5 \times 1.5 \mathrm{~cm})$ and the animals were sacrificed (Figure 5B). These results clearly demonstrate the tumor accumulation of $\mathrm{BPF}$ and its photodynamic ability to cause tumor regression. 

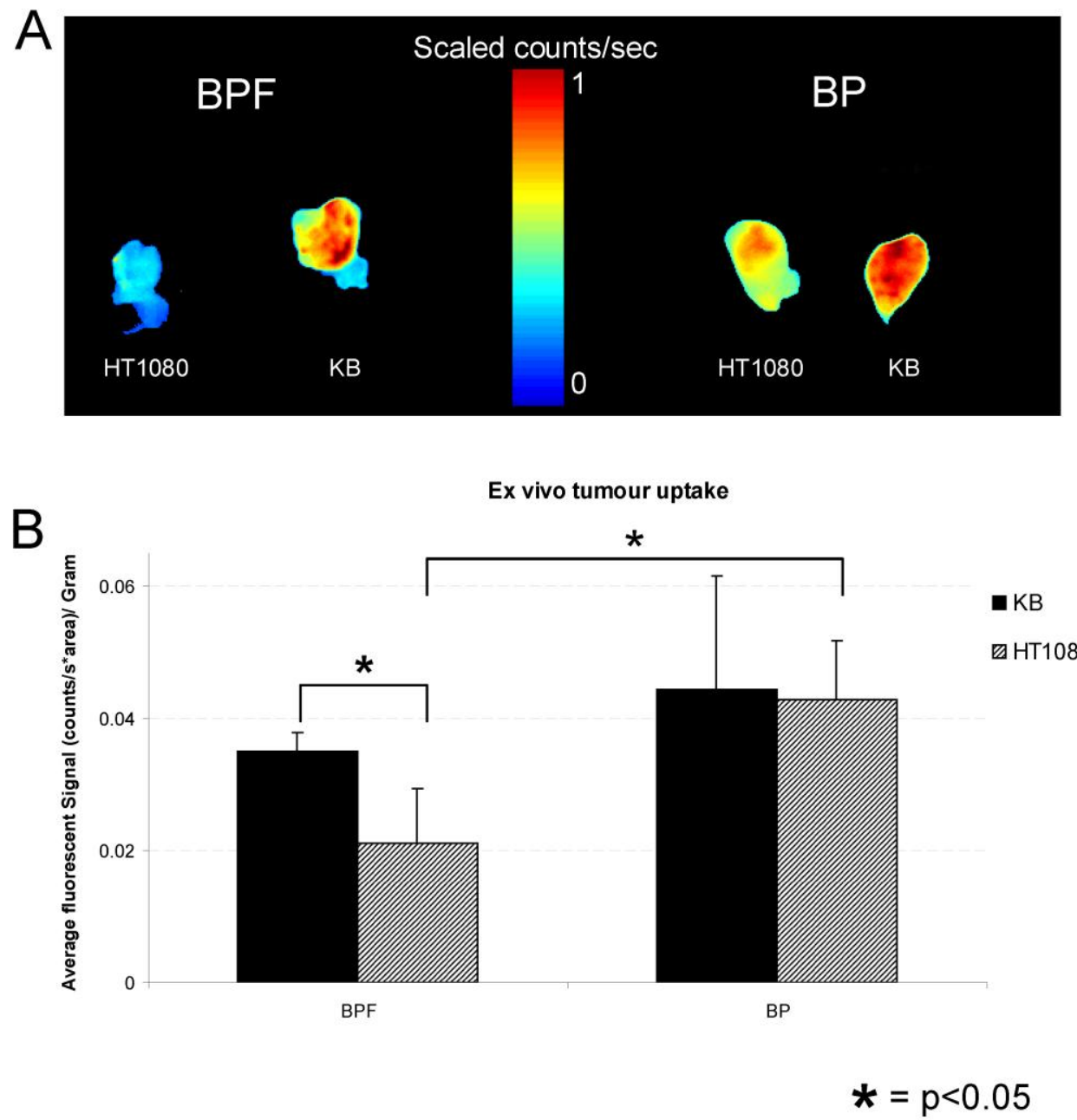

Figure 4. A) Ex vivo fluorescent imaging of excised $\mathrm{KB}$ and $\mathrm{HTI} 080$ tumors from animals administered with $25 \mathrm{nmol}$ of BPF or BP. B) Average fluorescent signal of ex vivo tumor uptake of BPF or BP in KB and HTI 080 xenografts. Data are expressed as mean values $+\operatorname{SEM}(n=5)$ where $*$ depicts statistical significance with a $p<0.05$.

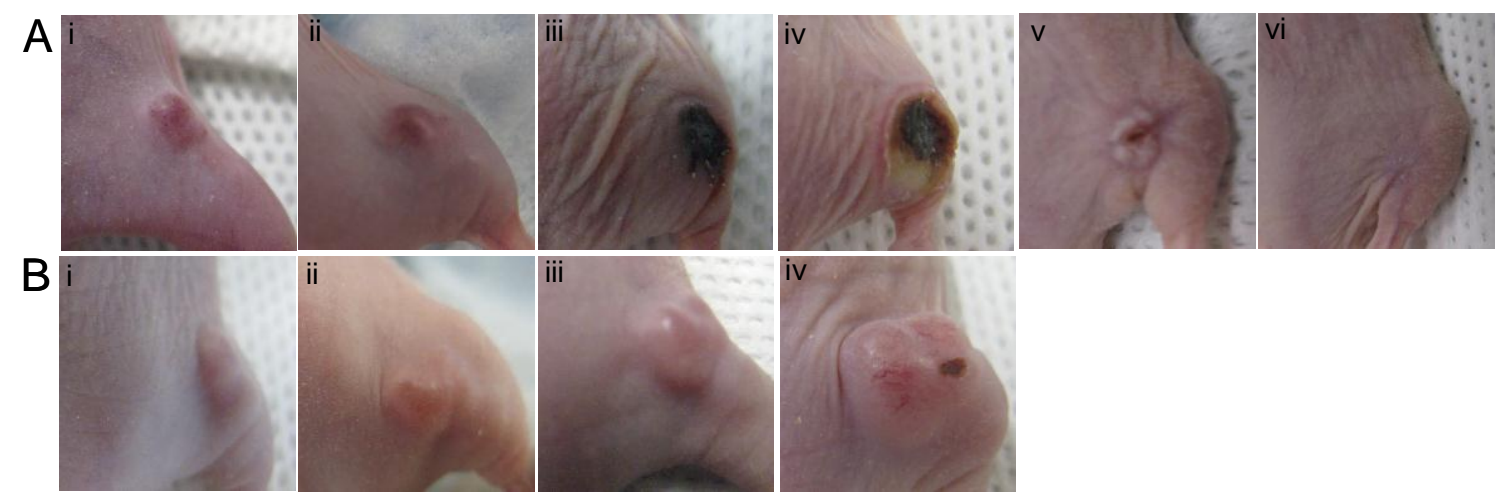

Figure 5. PDT efficacy in KB tumor-bearing mice (high FR expressing, right flank) $3 \mathrm{~h}$ after an intravenous injection of $A$ ) 50 nmol BPF or B) PBS (control) using a I 37 J PDT light dose at i) pre PDT, ii) 24h post PDT, iii) 2 days post PDT, iv) 7 days post PDT, v) 24 days post PDT, vi) 32 days post PDT. $(n=5)$ 


\section{Discussion}

We have successfully synthesized and characterized a novel targeted multimodality Bchl probe (BPF) with efficient optical imaging properties and effective PDT capabilities. In vivo optical imaging studies confirmed the FR targeting specificity of BPF and its ability to be retained and clearly delineate FR-expressing tumors. It is evident that the conjugation of folate to $\mathrm{BP}$ changes its properties and tumor retention characteristics. Folate deflects the potential of Bchl's own non-specific affinity to cancer cells. In vitro PDT studies demonstrated BPF's photocytotoxicity which was mirrored in vivo whereby complete tumor regression was achieved. Although a 16h drug-light interval was used in vitro, a $3 \mathrm{~h}$ drug-light interval was used in vivo as the optical imaging studies demonstrated that the highest BPF uptake occurred at $3 \mathrm{~h}$. This is not surprising as probe uptake demonstrated in vitro rarely translates to similar uptake characteristics in vivo.

Fluorescence imaging is proving to be a powerful tool for cancer diagnostics and treatment. It has received much attention due to its ease of implementation, operational simplicity, low cost and recent development of novel fluorescent probes [2, 4, 27]. The tumor targeting ability of BPF and its favorable excitation and emission spectra $\left(\lambda_{\text {excitation }}=748 \mathrm{~nm}\right.$, $\left.\lambda_{\text {emission }}=766 \mathrm{~nm}\right)$ make it an attractive optical agent providing efficient tissue penetration of photons and minimal background autofluorescence. The optical imaging properties of BPF with its tumor selectivity may enable its use as an effective image guidance tool during surgical resection. BPF better delineates tumor margins within the surgical bed, thereby ensuring complete tumor resection and aid in the intra-operative detection of small metastatic lesions that are otherwise not visible. BPF could also be used as means to 'clean up' the surgical bed following resection. PDT treatment of the entire surgical bed post-resection could result in the eradication of any residual tumor mass and microscopic metastatic deposits, while preserving normal tissues.

Recently, interest has risen in using positron emission tomography (PET) for image guided therapy. PET is attractive as it provides quantitative drug biodistribution, effective treatment planning and provides non-invasive deep tissue images, key characteristics not achievable by optical imaging and/or PDT [28]. Similar to optical probes, targeted radiotracer development is an important key to increasing tumor specificity and sensitivity [29]. Thus, attempts have been made to exploit the tumor avidity of porphyrins by the incorporation of radioisotopes for tar- geting PET probes [30-31]. However, as demonstrated by TOOKAD, Bchl itself is an excellent metal chelator forming a highly stable metallo-complex making it potentially an efficient delivery vehicle of radioisotopes. Of particular interest is copper-64 $\left({ }^{64} \mathrm{Cu}\right)$ [32] as $\mathrm{Cu}$-porphyrins are exceedingly stable to demetallation, has patient-tested non-toxicity, have comparable ${ }^{64} \mathrm{Cu}$ half-life with porphyrin pharmacokinetics and most importantly, ${ }^{64} \mathrm{Cu}$-chelation does not alter the biodistribution of its host porphyrin [33-38]. Previous studies have demonstrated that the stability of Bchl is dependent on its central metal where the $\mathrm{Cu}-\mathrm{Bchl}$ metal complex is the most stable [19]. Thus, similar to how palladium stabilizes Bchl (TOOKAD), the insertion of ${ }^{64} \mathrm{Cu}$ into Bchl's porphyrin ring may further stabilize Bchl making ${ }^{64} \mathrm{Cu}$-BPF not only an effective, but highly stable, PET imaging probe. Preliminary experiments evaluated the feasibility of radiolabeling BPF with ${ }^{64} \mathrm{Cu}$, Supplementary Material: Figure S2. Although we were able to radiolabel BPF with ${ }^{64} \mathrm{Cu}$ following ${ }^{64} \mathrm{Cu}$-porphyrin labeling procedures that we recently reported [39], within $24 \mathrm{~h}$, some demetallation was observed, indicating an unstable metallporphyrin complex. Further studies evaluating different radiochemistry methods for a more stable chelation of ${ }^{64} \mathrm{Cu}$ to Bchl are currently underway. We also preliminarily explored the potential of ${ }^{64} \mathrm{Cu}-\mathrm{BPF}$ to accumulate and image FR positive tumors (Supplementary Material: Figure S3). As the radiolabeling chemistry of ${ }^{64} \mathrm{Cu}-\mathrm{BPF}$ needs to be further evaluated to ensure stable ${ }^{64} \mathrm{Cu}$ chelation, PET imaging studies did not demonstrate ideal tumor delineation, however, some ${ }^{64} \mathrm{Cu}-\mathrm{BPF}$ tumor accumulation was observed (Supplementary Material: Figure S3). The appeal of introducing PET properties into BPF addresses many limitations currently faced by optical imaging including unlimited deep tissue penetration, quantitative biodistribution and an efficient non-invasive means for treatment planning. The complementary nature of PET and optical imaging may provide a means to translate PET treatment planning to the surgical table using fluorescence image guidance with a single probe.

There are several advantages to having multimodal applications in a single probe as it ensures the same pharmacokinetics and colocalization of the probe for all applications. This is highly appealing especially when therapy is involved as additional stress on the body's blood clearance mechanisms is avoided which often accompanies administration of multiple doses of agents [27]. Thus, the use of a single agent dismisses any variability in the tumor uptake specificity, pharmacokinetics and pharmacodynamics of BPF. Small molecule multimodal probes, such as $\mathrm{BPF}$, are attractive because they are typically cleared 
by the renal system avoiding any toxicity associated with long-term liver retention, have a fast clearance time and rapid diffusion through tissue $[27,40]$. Sensitivities for radiotracers and optical imaging probes are within the same range [41] and therefore, an attractive combination. The multimodal nature of BPF provides complimentary information with regards to tumor delineation useful in image guidance as well as drug accumulation, pharmacokinectics and pharmacodynamics which is particularly useful in determining the ideal drug-light interval in PDT treatments. This efficient PDT capability of BPF combined with its optical and potential PET imaging properties makes BPF a powerful imaging and therapeutic tool that may boost the clinical benefits of hybrid technologies.

\section{Conclusion}

Here we report the successful synthesis and characterization of a novel multimodal FR targeted optical imaging and PDT agent. All results demonstrate the FR specificity of BPF as it only accumulates in FR expressing tumors in vivo and serves as an efficient fluorescent imaging probe for the detection of FR positive tumors. Furthermore, BPF caused complete tumor regression in vivo after PDT treatment. Therefore, BPF is not only a specific diagnostic probe for FR expressing tumors but also a promising therapeutic agent for FR positive cancers. BPF provides a more sensitive tumor detection strategy and its PDT therapeutic ability provides a "see and treat" approach ensuring specific treatment of targeted sites. FR is overexpressed in a variety of epithelial cancers particularly in ovarian, head and neck, brain, breast, colon, renal and lung carcinoma with limited expression in normal tissues [42-43]. In addition to the promise of FR as an oncologic biomarker, FR is also overexpressed and accessible on activated macrophages (i.e. associated with many inflammatory diseases) [42-43]. BPF may play an important role in the imaging and therapy of FR positive diseases such as rheumatoid arthritis, Crohn's disease, atherosclerosis, and organ transplant rejection [42]. We envision that BPF may be potentially useful for disease detection, image-guided surgery allowing completeness of resection by "extending the surgeon's vision" and/or with adjuvant PDT treatment to safely and effectively eradicate any post-resection residual tumor cells and eliminate microscopic deposits. Finally, the folate homing molecule in BPF may be switched to another disease specific biomarker providing a skeleton for numerous targeted multifunctional Bchl probes that would allow for earlier diagnosis and more effective therapeutics.

\section{Acknowledgements}

Financial support for this work was provided by the Ontario Institute for Cancer Research, the Canadian Institute of Health Research, the DOD BCRP Predoc Award W81XWH-10-1-0115, the Natural Sciences and Engineering Research Council of Canada, the Canadian Foundation of Innovation, and the Joey and Toby Tanenbaum/Brazilian Ball Chair in Prostate Cancer Research.

\section{Supplementary Material}

Concentration dependent in vitro PDT cell killing, trimodality design (fluorescence, PDT and PET imaging), ${ }^{64} \mathrm{Cu}$ radiolabeling of BPF and preliminary PET imaging study. http://www.thno.org/v0 I p0354s I.pdf

\section{Conflict of Interest}

The authors have declared that no conflict of interest exists.

\section{References}

1. Chen XS. Introducing Theranostics Journal - From the Editor-in-Chief. Theranostics. 2011; 1: 1-2.

2. Weissleder R, Pittet MJ. Imaging in the era of molecular oncology. Nature. 2008; 452: 580-9.

3. Moriyama EH, Zheng G, Wilson BC. Optical molecular imaging: from single cell to patient. Clin Pharmacol Ther. 2008; 84: 267-71.

4. Celli JP, Spring BQ, Rizvi I, Evans CL, Samkoe KS, Verma S, et al. Imaging and photodynamic therapy: mechanisms, monitoring, and optimization. Chem Rev. 2010; 110: 2795-838.

5. Lovell JF, Liu TW, Chen J, Zheng G. Activatable photosensitizers for imaging and therapy. Chem Rev. 2010; 110: 2839-57.

6. Liu TW, Chen J, Zheng G. Peptide-based molecular beacons for cancer imaging and therapy. Amino Acids. 2010; epub.

7. Stefflova K, Chen J, Zheng G. Killer beacons for combined cancer imaging and therapy. Curr Med Chem. 2007; 14: 2110-25.

8. Dougherty TJ, Gomer CJ, Henderson BW, Jori G, Kessel D, Korbelik M, et al. Photodynamic therapy. J Natl Cancer Inst. 1998; 90: 889-905.

9. Wilson BC, Patterson MS. The physics, biophysics and technology of photodynamic therapy. Phys Med Biol. 2008; 53: R61-109.

10. Lo PC, Chen J, Stefflova K, Warren MS, Navab R, Bandarchi B, et al. Photodynamic molecular beacon triggered by fibroblast activation protein on cancer-associated fibroblasts for diagnosis and treatment of epithelial cancers. J Med Chem. 2009; 52: 358-68.

11. Zheng G, Chen J, Stefflova K, Jarvi M, Li H, Wilson BC. Photodynamic molecular beacon as an activatable photosensitizer based on protease-controlled singlet oxygen quenching and activation. Proc Natl Acad Sci U S A. 2007; 104: 8989-94.

12. Stefflova K, Chen J, Li H, Zheng G. Targeted photodynamic therapy agent with a built-in apoptosis sensor for in vivo near-infrared imaging of tumor apoptosis triggered by its photosensitization in situ. Mol Imaging. 2006; 5: 520-32.

13. Jeong H, Huh M, Lee SJ, Koo H, Kwon IC, Jeong SY, et al. Photosensitizer-conjugated human serum albumin 
nanoparticles for effective photodynamic therapy. Theranostics. 2011; 1: 230-9.

14. Pandey RK, Goswami LN, Chen Y, Gryshuk A, Missert JR, Oseroff A, et al. Nature: a rich source for developing multifunctional agents. Tumor-imaging and photodynamic therapy. Lasers Surg Med. 2006; 38: 445-67.

15. Cao W, Ng KK, Corbin I, Zhang Z, Ding L, Chen J, et al. Synthesis and evaluation of a stable bacteriochlorophyll-analog and its incorporation into high-density lipoprotein nanoparticles for tumor imaging. Bioconjug Chem. 2009; 20: 2023-31.

16. Chen Y, Graham A, Potter W, Morgan J, Vaughan L, Bellnier DA, et al. Bacteriopurpurinimides: highly stable and potent photosensitizers for photodynamic therapy. J Med Chem. 2002; 45: 255-8.

17. Rosenbach-Belkin V, Chen L, Fiedor L, Tregub I, Paviotsky F, Brumfeld V, et al. Serine conjugates of chlorophyll and bacteriochlorophyll: photocytotoxicity in vitro and tissue distribution in mice bearing melanoma tumors. Photochem Photobiol. 1996; 64: 174-81.

18. Gryshuk AL, Chen Y, Potter W, Ohulchansky T, Oseroff A, Pandey RK. In vivo stability and photodynamic efficacy of fluorinated bacteriopurpurinimides derived from bacteriochlorophyll-a. J Med Chem. 2006; 49: 1874-81.

19. Fiedor J, Fiedor L, Kammhuber N, Scherz A, Scheer H. Photodynamics of the bacteriochlorophyll-carotenoid system. 2 . Influence of central metal, solvent and beta-carotene on photobleaching of bacteriochlorophyll derivatives. Photochem Photobiol. 2002; 76: 145-52.

20. Marotta DE, Cao W, Wileyto EP, Li H, Corbin I, Rickter E, et al. Evaluation of bacteriochlorophyll-reconstituted low-density lipoprotein nanoparticles for photodynamic therapy efficacy in vivo. Nanomedicine (Lond). 2011; 6: 475-87.

21. Trachtenberg J, Bogaards A, Weersink RA, Haider MA, Evans A, McCluskey SA, et al. Vascular targeted photodynamic therapy with palladium-bacteriopheophorbide photosensitizer for recurrent prostate cancer following definitive radiation therapy: assessment of safety and treatment response. J Urol. 2007; 178: 1974-9.

22. Trachtenberg J, Weersink RA, Davidson SR, Haider MA, Bogaards A, Gertner MR, et al. Vascular-targeted photodynamic therapy (padoporfin, WST09) for recurrent prostate cancer after failure of external beam radiotherapy: a study of escalating light doses. BJU Int. 2008; 102: 556-62.

23. Stefflova K, Li H, Chen J, Zheng G. Peptide-based pharmacomodulation of a cancer-targeted optical imaging and photodynamic therapy agent. Bioconjug Chem. 2007; 18: 379-88.

24. Schreiber S, Gross S, Brandis A, Harmelin A, Rosenbach-Belkin $\mathrm{V}$, Scherz A, et al. Local photodynamic therapy (PDT) of rat C6 glioma xenografts with Pd-bacteriopheophorbide leads to decreased metastases and increase of animal cure compared with surgery. Int J Cancer. 2002; 99: 279-85.

25. Brun PH, DeGroot JL, Dickson EF, Farahani M, Pottier RH. Determination of the in vivo pharmacokinetics of palladium-bacteriopheophorbide (WST09) in EMT6 tumor-bearing Balb/c mice using graphite furnace atomic absorption spectroscopy. Photochem Photobiol Sci. 2004; 3: 1006-10.

26. Koudinova NV, Pinthus JH, Brandis A, Brenner O, Bendel P, Ramon J, et al. Photodynamic therapy with Pd-Bacteriopheophorbide (TOOKAD): successful in vivo treatment of human prostatic small cell carcinoma xenografts. Int J Cancer. 2003; 104: 782-9.

27. Louie A. Multimodality imaging probes: design and challenges. Chem Rev. 2010; 110: 3146-95.

28. Gambhir SS. Molecular imaging of cancer with positron emission tomography. Nat Rev Cancer. 2002; 2: 683-93.
29. Chen X. Integrin Targeted Imaging and Therapy. Theranostics. 2011; 2011: 28-9.

30. Pandey SK, Gryshuk AL, Sajjad M, Zheng X, Chen Y, Abouzeid $\mathrm{MM}$, et al. Multimodality agents for tumor imaging (PET, fluorescence) and photodynamic therapy. A possible "see and treat" approach. J Med Chem. 2005; 48: 6286-95.

31. Pandey SK, Sajjad M, Chen Y, Pandey A, Missert JR, Batt C, et al. Compared to purpurinimides, the pyropheophorbide containing an iodobenzyl group showed enhanced PDT efficacy and tumor imaging (124I-PET) ability. Bioconjug Chem. 2009; 20: 274-82.

32. Jacobson O, Weiss ID, Szajek LP, Niu G, Ma Y, Kiesewetter DO, et al. PET imaging of CXCR4 using copper-64 labeled peptide antagonist. Theranostics. 2011; 1: 251-62.

33. Bases R, Brodie SS, Rubenfeld S. Attempts at tumor localization using $\mathrm{Cu}$ 64-labeled copper porphyrins. Cancer. 1958; 11: 259-63.

34. Firnau G, Maass D, Wilson BC, Jeeves WP. 64Cu labelling of hematoporphyrin derivative for non-invasive in-vivo measurements of tumor uptake. Prog Clin Biol Res. 1984; 170: 629-36.

35. Hambright P, Fawwaz R, Valk P, McRae J, Bearden AJ. The distribution of various water soluble radioactive metalloporphyrins in tumor bearing mice. Bioinorg Chem. 1975; 5: 87-92.

36. Policard A. Etudes sur les aspects offerts par des tumeurs experimentales examinees a la lumiere des Woods. Compt Rend Soc Biol. 1924; 91: 1423.

37. Wilson BC, Firnau G, Jeeves WP, Brown KL, Burns-McCormick DM. Chromatographic analysis and tissue distribution of radiocopper-labelled haematoporphyrin derivatives. Laser Med Sci. 1988; 3: 71-80.

38. Wrenn FRJr., Good ML, Handler P. The use of positron-emitting radioisotopes for the localization of brain tumors. Science. 1951; 113: 525-7.

39. Shi J, Liu TW, Chen J, Green D, Jaffray D, Wilson BC, et al. Transforming a targeted porphyrin theranostic agent into a PET imaging probe for cancer. Theranostics. 2011; to appear.

40. Zhang Y, Yang Y, Cai W. Multimodality Imaging of Integrin alpha(v)beta(3) Expression. Theranostics. 2011; 1: 135-48.

41. Zhang F, Zhu L, Liu G, Hida N, Lu G, Eden HS, et al. Multimodality imaging of tumor response to doxil. Theranostics. 2011; 1: 302-9.

42. Low PS, Kularatne SA. Folate-targeted therapeutic and imaging agents for cancer. Curr Opin Chem Biol. 2009; 13: 256-62.

43. Leamon CP, Jackman AL. Exploitation of the folate receptor in the management of cancer and inflammatory disease. Vitam Horm. 2008; 79: 203-33. 